\title{
CAAI-a cognitive architecture to introduce artificial intelligence in cyber-physical production systems
}

\author{
Andreas Fischbach $^{1}$ (D) . Jan Strohschein ${ }^{2} \cdot$ Andreas Bunte $^{3} \cdot$ Jörg Stork $^{1} \cdot$ Heide Faeskorn-Woyke $^{2} \cdot$ Natalia Moriz $^{3}$. \\ Thomas Bartz-Beielstein ${ }^{1}$
}

Received: 28 February 2020 / Accepted: 14 September 2020 / Published online: 1 October 2020

(c) The Author(s) 2020

\begin{abstract}
This paper introduces CAAI, a novel cognitive architecture for artificial intelligence in cyber-physical production systems. The goal of the architecture is to reduce the implementation effort for the usage of artificial intelligence algorithms. The core of the CAAI is a cognitive module that processes the user's declarative goals, selects suitable models and algorithms, and creates a configuration for the execution of a processing pipeline on a big data platform. Constant observation and evaluation against performance criteria assess the performance of pipelines for many and different use cases. Based on these evaluations, the pipelines are automatically adapted if necessary. The modular design with well-defined interfaces enables the reusability and extensibility of pipeline components. A big data platform implements this modular design supported by technologies such as Docker, Kubernetes, and Kafka for virtualization and orchestration of the individual components and their communication. The implementation of the architecture is evaluated using a real-world use case. The prototypic implementation is accessible on GitHub and contains a demonstration.
\end{abstract}

Keywords CPPS · Artificial intelligence $\cdot$ Industry 4.0 $\cdot$ Reference architecture $\cdot$ Optimization $\cdot$ SMBO $\cdot$ Cognition . Big data platform $\cdot$ Modularization $\cdot$ AutoML

Andreas Fischbach

andreas.fischbach@th-koeln.de

Jan Strohschein

jan.strohschein@th-koeln.de

Andreas Bunte

andreas.bunte@th-owl.de

Jörg Stork

joerg.stork@th-koeln.de

Heide Faeskorn-Woyke

heide.faeskorn-woyke@th-koeln.de

Natalia Moriz

natalia.moriz@th-owl.de

Thomas Bartz-Beielstein

thomas.bartz-beielstein@th-koeln.de

1 TH Köln, Institute for Data Science, Engineering, and Analytics, Gummersbach, Germany

2 TH Köln, Institute of Computer Science, Gummersbach, Germany

3 Institute Industrial IT, OWL University of Applied Sciences and Arts, Lemgo, Germany

\section{Introduction}

The use of artificial intelligence (AI) in cyber-physical production systems (CPPS) can help to significantly reduce costs and provides new market opportunities [21]. Many Industry 4.0 (I4.0) applications rely on the use of AI, such as condition monitoring, predictive maintenance, diagnosis, or optimization $[6,13]$. Until now, the implementation of real-world use cases is time- and cost-intensive due to missing standards and imprecisely described architectures. Typically, an AI expert analyzes one specific application and develops a suitable solution that will match customer needs. Often use cases, particularly those in small and medium-sized enterprise (SMEs), are not implemented because of limited resources and the unpredictable benefit of AI solutions. Therefore, to enable a resource-efficient use for many applications, AI solutions require moderate manual implementation and operation effort.

Goals (G) for the cognitive architecture for artificial intelligence in cyber-physical production systems $(\mathrm{CAAI})^{1}$

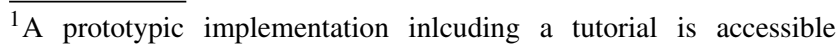
on GitHub: https://github.com/janstrohschein/KOARCH/tree/master/ Use_Cases/VPS_Popcorn_Production/Docker
} 
application are represented by several SME requirements that have to be fulfilled by an AI solution.

(G-1) Reliability: In a competitive market environment, the efficiency of CPPS is important, and reliability is a prerequisite to achieving it. Since the CAAI supports the CPPS, both are interconnected and share the same requirements. Capturing the complete data is essential. It contains a vast amount of value, especially if it includes information about the quality of produced products, which can be utilized by applications of AI. To avoid losses of data or downtime of the CPPS, the CAAI and its underlying infrastructure have to be stable and reliable $[12,41]$.

(G-2) Flexibility: A significant drawback of existing AI solutions is that an AI expert often develops them for a single machine or a single problem. Therefore, these solutions do not include common interfaces, which enable an adaption or extension of the existing system. This inflexibility is not acceptable, because there is a great demand in the market for fast adapting CPPS, which can not be fulfilled by the approach of specific AI solutions. The CAAI has to be flexible and extendable to enable a quick reaction on this market demand [10].

(G-3) Generalizability: The CAAI should apply to many types of CPPS and support many different use cases. As it is impossible to choose algorithms for all system type combinations and applications in advance, their selection should be performed automatically in an intelligent manner. Thus, the CAAI has to process the user-defined aims, derive a valuable process pipeline for the specific system, and learn over time to improve the system's performance, i.e., the CAAI implements cognitive capabilities [36].

(G-4) Adaptability: The realization of adaptability through the CAAI increases the efficiency of the CPPS by directly adjusting process parameters so that users do not have to change them manually. Furthermore, adaptability allows us to automate the adjustment, which is less error-prone, and ultimately realize an autonomous system. However, the CAAI has to ensure the safe operation of the CPPS during the whole process. For example, the operation boundaries of the CPPS have to be respected during optimization. In contrast, in anomaly detection, there might be machine parts that need to proceed operation, even in case of emergency. Thus, the boundary conditions must be included in the CAAI and CPPS adjustments are only allowed within these boundaries [27, 36].
First, we review existing approaches. They are associated with the goals (G1-G4). To the best of our knowledge, until now, no work tackles those goals properly. Proposed architectures from the domain of automation, i.e., the Reference Architecture Model Industrie 4.0 (RAMI4.0) [1], the Industrial Internet Reference Architecture (IIRA) [29], or the 5C architecture [26], are too abstract since they do not define implementation details, such as interfaces. It is necessary to extend and refine certain parts of them to achieve a more specific architecture. Cognitive architectures, such as Adaptive Control of Thought-Rational (ACT-R) [2] and Soar [25], implement certain concepts to reach adaption and cognitive capabilities.

They can not be directly used to address industrial use cases, because they focus on cognition and lack generality [8]. Automated Machine Learning (AutoML) [14] and hyperheuristics can choose and configure a suitable algorithm automatically. That includes steps such as data pre-processing, algorithm selection, and hyperparameter optimization [15, 35, 45]. Since there is an intersection between our architecture and these methods, they are considered for our implementation. Some Big Data Platform (BDPs) can be found in the literature, such as the Open Big Data Platform for Industry 4.0 [47], the Big Data Analytics Architecture for Industry 4.0 [39], and the Big Data for Industry 4.0 [16]. To the best of our knowledge, none of these BDP fulfills all our requirements towards a cognitive architecture.

According to Neisser [34], cognition refers to "all processes by which the sensory input is transformed, reduced, elaborated, stored, recovered, and used". Regarding the context of I4.0, in the scope of CAAI we define cognition as follows.

Definition 1 (Cognition) Cognition refers to all processes that transform, reduce, elaborate, store, recover, and use input data to solve I4.0 use cases, i.e., condition monitoring, anomaly detection, optimization, and predictive maintenance.

The central part of CAAI is a cognitive module that processes knowledge, interprets aims, creates appropriate pipelines, and improves the system through continuous evaluation. According to (G-2), our architecture is developed as an extensible platform, to integrate future developments of AutoML related methods. Additionally, the cognition module stores a priori knowledge that is valid for all use cases, e.g., information about suitable algorithms to solve specific tasks such as multi-criteria optimization or time series anomaly detection. By learning from experience, the knowledge grows, and the performance of the system improves over time. Due to its modularity, the architecture is extensible and allows the integration of new algorithms into the 
CAAI. Therefore, technologies such as containerization, messaging, and well-defined interfaces are used to achieve an abstracted and language-agnostic view and thus encapsulate the algorithms in separated modules. Furthermore, the architecture enables an adaption of the CPPS if a promising configuration was determined. Even though it is difficult for CAAI to reach results that are equivalent to a customized solution from an AI expert, it will achieve improvements in a cost-efficient manner for many use cases, without the need of support from an AI expert.

Depending on the individual problem characteristic, which changes from use case to use case, some algorithms might be superior to others in terms of either performance or computation time. Additionally, each algorithm needs several pre-processing steps, e.g., feature creation, feature selection, or model building. The type of models, their parameter values and the algorithm topologies can be learned, at least to some extent, by the system itself.

This paper's main contribution is a novel cognitive architecture for CPPS, which has several advantages in comparison with state-of-the-art architectures. To tackle goals (G-1) to (G-4), the following methods (M) are considered in this paper:

(M-1) Big Data Platform: Continuous and reliable operation (G-1) is ensured by a BDP. The CAAI-Big Data Platform (CAAI-BDP) comprises different techniques to reach the goal, such as orchestration, virtualization, and containerization. The orchestration instantiates and connects the selected modules and thus creates the pipelines needed for the processing. Furthermore, the orchestration enables to move applications and their respective containers to the remaining infrastructure if certain parts of the system fail. Containerization, which provides virtualization on operating-system-level, allows the existence of multiple isolated user-space instances. It improves reliability because each instance can only access its container's contents and devices assigned to this container [33]. Orchestration of virtualized containers also aids scalability as it is possible to create several instances of a container to work in parallel.

(M-2) Modularization: A modularization of the AI components enables the flexibility (G-2) of the CAAI to adapt it to specific requirements. Moreover, modular components require well-specified interfaces with detailed definitions. Furthermore, the modular design reduces development and maintenance costs by the integration of existing components. Thus, only new components have to be developed.

(M-3) Cognition: Automated process pipeline generation methods enable the realization of different use cases without the involvement of an AI expert and the transfer to different types of CPPS. The automated pipeline creation is an important feature to ensure generalizability (G-3) for specific use cases, dynamic systems, and changing environments. It is realized by the cognitive module, which generates the pipeline and selects the best fitting algorithms according to the given data and defined goal. The pipeline evaluation is automated to improve results and detect performance drifts. The cognitive module subsequently evaluates the various pipelines to collect information about the performance of different algorithms for a given use case. This evaluation enables learning over time, and the detection of performance drifts that may result in a re-calibration of the processing pipeline.

(M-4) Automatic Decision: The algorithm's results can be interpreted, and suggestions for an adaption can be derived, enabling automatic or CAAI supported adaptions of the CPPS. Therefore, new parameter sets or other system changes, identified by the algorithms, have to be applied to the CPPS (G-4). Furthermore, boundary conditions can be defined and applied for decision making, e.g., a minimum of expected improvement or specifications that ensure a safe adaption. Moreover, the decision must be applied to the CPPS controller, which adapts the physical machine. For realization, several existing approaches, such as skill-based engineering, can be used [10, 30, 49].

The remainder of this paper is organized as follows: Section 2 introduces the proposed cognitive architecture. A real-world use case evaluates the CAAI architecture as described in Section 3 along with available techniques to implement the methods, design choices, and the results of our implementation. Finally, the conclusion and outlook Section 4 presents our major findings and resulting future research tasks.

\section{The CAAI architecture}

In this section, we introduce the CAAI, which aims to have a reference character, on a rather abstract level. Detaching the description from the concrete implementation allows choosing implementation technologies according to individual preferences or technological advances. Nevertheless, Section 3 introduces an exemplary implementation of the CAAI. The architecture addresses the methods (M-1) to (M-4) to reach the goals (G-1) to (G-4). 


\subsection{Overview}

Our cognitive architecture builds upon modeling the information, data applications, and streams required for specific tasks in the I4.0 scenario while providing reliability, flexibility, generalizability, and adaptability. The concept, depicted in Fig. 1, is based on a three-tier architecture to simplify interoperability and to ensure horizontal scalability.

The CAAI approach addresses sub-symbolic AI. In contrast to symbolic AI, sub-symbolic AI does not use human-readable problem representations. Sub-symbolic AI, such as neural networks and deep learning, perform calculations based on principles that have demonstrated to be able to solve problems.

The CAAI-CBDP wraps the architecture and arranges software modules in two processing layers, the data processing layer (DPL), and the conceptual layer (CL) and connects them via three bus systems (data, analytics, and knowledge bus). Data from a CPPS enters the system at the very bottom (see Fig. 1). The protocol translation module transforms incoming data and sends it to the data bus. The pre-processing module receives the raw data, performs the necessary steps to clean the data, and publishes the results back to the data bus. Other modules in the data processing layer utilize data from the data bus and transfer their analytical results onto the analytics bus. Modules in the CL process information about the user-defined aims and the business logic for a given use case. They evaluate the results from the analytics bus, determine the parameters to adjust the CPPS via the adaption module, and measure the overall system performance. The CL modules also interact with the knowledge bus to generate reports for the user and process new instructions.

The central element of the architecture is the cognitive module, which selects and orchestrates different analyses and algorithms, depending on the use case. Therefore, the composition of active modules and their communication over the bus system will change during run time. Providing a pre-defined set of modules and the capability to add new modules reduces the overall implementation complexity by building a cohesive yet modular solution. The following sections describe the bus infrastructure, the layers, and the cognitive module in more detail.

\subsection{Bus infrastructure}

Different modules of the system communicate asynchronously via three buses, the data bus, analytics bus, and knowledge bus. The processing degree of the data grows
Fig. 1 CAAI architecture overview. The CAAI-BDP, depicted in dark gray, manages the bus systems and layers. Bus systems are colored in blue and establish communication between the modules. The arrows demonstrate the designated information flow. The layers are shaded in light gray and contain the different modules. The cognition module, which establishes automatic configuration, is colored in turquoise. Oval shapes depict external systems, e.g., a CPPS or a Human-Machine Interface

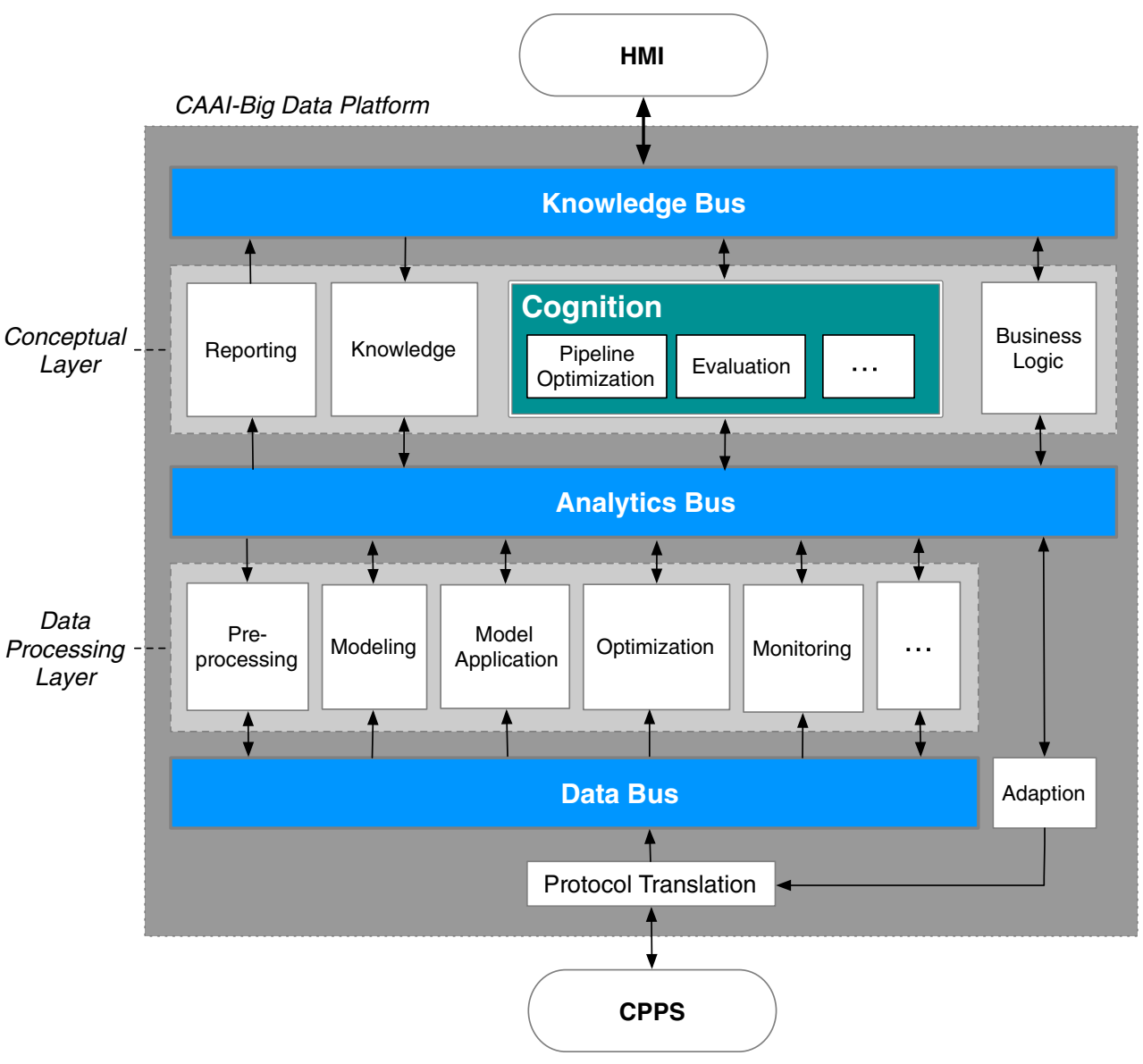


incrementally from bottom to top, and additionally in some cases horizontally as well. Each bus covers several topics, which can be subscribed by modules attached to the bus. All modules publish their data to the relevant attached bus on a pre-defined topic, so one or more other modules can use the data and intermediate results for their processing. Thus, the architecture implements a message-driven processing approach, leading to a flexible and agile system with clear interfaces and hierarchies. The main features of applications that use message queuing techniques are [18]:

(i) no direct connections between modules,

(ii) communication between modules can be independent of time,

(iii) work can be carried out by small, self-contained modules,

(iv) communication can be driven by events,

(v) data integrity through validation schemata, and

(vi) recovery support.

The following paragraphs give a detailed description of the three bus systems, while Table 1 presents an overview and summarizes the differences of the respective data type.

\subsubsection{Data bus}

The data bus transports raw data from a CPPS, as well as data from demonstrators, external simulators, or simulation modules. Cleaned and further pre-processed data is also published back to the data bus by the pre-processing modules. Therefore, the data volume and velocity are high, even though the entropy is still quite low, and interpretability is complicated. Entropy describes the average level of information in any new message in the context of information theory [42]. The content in messages coming from the CPPS is often quite similar; therefore, the entropy is accordingly low. Overall the data bus transports streams of real-time data.

\subsubsection{Analytics bus}

Aggregated data and intermediate results transported on the analytics bus have a higher information density than data from the data bus. The number of processing pipelines and the type of used algorithms determines the computational effort. These get instantiated by the cognition module and are expected to use a significant amount of the available processing power. Consequently, the analytics bus distributes knowledge, models, and results from the model application, the monitoring module, and the business logic to derive commands to adjust the system.

\subsubsection{Knowledge bus}

The knowledge bus enables the communication between the end-user and the system and combines the knowledge, business logic, and user-defined goals and actions. The cognitive module receives declarative goals defined by the user [8]. Information from the analytics bus condenses into reports for the user. Furthermore, feedback from the user can be requested. So the knowledge bus uses enriched data, which aids the interpretability and provides the most value for the user.

\subsection{Layer}

Several modules process the data within two layers. Each layer can be extended individually. Each of the modules processes the data in a specific manner, e.g., with a specific algorithm. Several modules are combined to enable complex data processing.

\subsubsection{Data processing layer}

The DPL contains all modules that are processing subsymbolic data. Instances of modules are combined in processing pipelines in order to solve the desired task based on the raw data (see Fig. 2). Except for the monitoring module, all initially provided modules belong to one of the following three types:

(i) Pre-processing modules receive data from the data bus and provide results to the data bus. They prepare data for the usage, e.g., by imputing missing values or synchronizing time stamps.

Table 1 Comparison of the data on the three bus systems used in the CAAI

\begin{tabular}{llll}
\hline & Data bus & Analytics bus & Knowledge bus \\
\hline Type of data & Raw, pre-processed & Processed & Enriched \\
Volume and Velocity & High & Moderate & Low \\
Computational effort & Low & High & Moderate \\
Entropy & Low & Moderate & High \\
Interpretability & Complicated & Moderate & Easy \\
\hline
\end{tabular}


Fig. 2 Top: DPL with modules highlighted that can be selected and varied by the cognitive module. Bottom: An example of four different process pipeline candidates. Two different modeling Algorithms (M1, M2) with different parameter setups (Setup A1, A2, B1, B2) and four different Modeling Application modules (MA1 to MA4) are used. Here, all pipelines use shared results of one pre-processing module

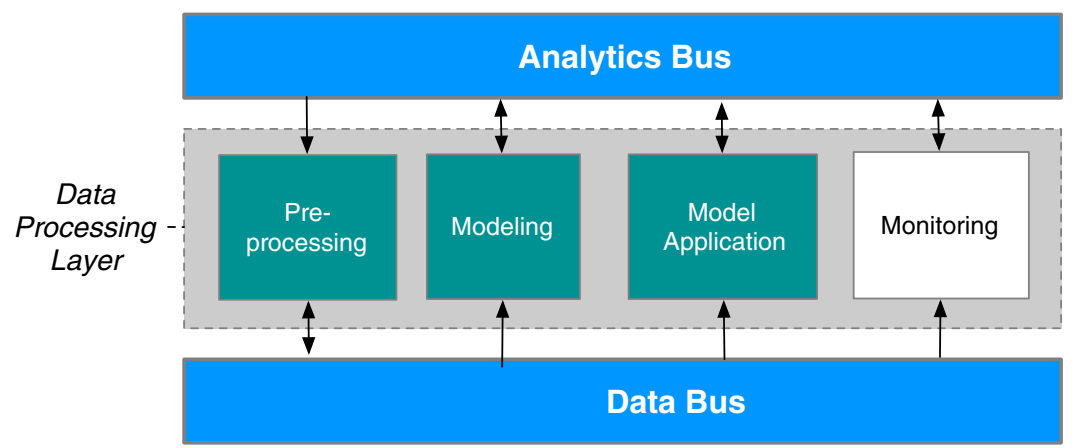

\begin{tabular}{|c|c|c|c|}
\hline Pipeline 1 & Preprocessing 1 & $\begin{array}{l}\text { Modeling M1 } \\
\text { Setup A1 }\end{array}$ & Model Application MA1 \\
\hline Pipeline 2 & Preprocessing 1 & $\begin{array}{l}\text { Modeling M1 } \\
\text { Setup A2 }\end{array}$ & Model Application MA2 \\
\hline Pipeline 3 & Preprocessing 1 & $\begin{array}{c}\text { Modeling M2 } \\
\text { Setup B1 }\end{array}$ & Model Application MA3 \\
\hline Pipeline 4 & Preprocessing 1 & $\begin{array}{l}\text { Modeling M2 } \\
\text { Setup B2 }\end{array}$ & Model Application MA4 \\
\hline
\end{tabular}

(ii) Modeling modules receive pre-processed data from the data bus and send their results to the analytics bus.

(iii) Model application modules get data from the data bus and the analytics bus and send their results to the analytics bus.

The modeling modules contain different machine learning algorithms in a modular manner. There is a need that the architecture incorporates multiple algorithms to chose appropriate modules, based on the task, the type of data, and the resulting model. It is also possible that modeling modules integrate expert knowledge into the model and provide tools for data curation. Model application modules can access the final model on the analytics bus. Additionally, the model application will access the data bus to compare the model with the process data to detect deviations, which are provided to the analytics bus. The cognitive module ensures that each model application module is compatible with a specific task and a particular model. Each of these components has a particular purpose, such as condition monitoring, predictive maintenance, diagnosis, optimization, or similar tasks.

\subsubsection{Conceptual layer}

The CL is located between the analytics bus and the knowledge bus and contains the following four modules.

(i) The reporting module visualizes the process data for the Human-Machine Interaction (HMI). It processes the data resulting from, e.g., monitoring or model application results.

(ii) The knowledge module contains

(a) relevant information about the CPPS, such as signal names, types of devices, or its topology,

(b) general knowledge, such as an algorithms topology, which describes the ability and properties of algorithms, and

(c) constraints that can be defined by the user, e.g., time constraints.

(iii) The business logic module decides whether an action is required or not. Therefore, it monitors the results from the model application modules, checks the constraints from the knowledge module, and derives actions, e.g., an adaption of the CPPS when a certain threshold is reached.

(iv) The cognition module is responsible for pipeline creation and optimization. If a specific task is provided by the user, the cognitive module aggregates and configures suitable modules of the DPL to fulfill the task. Hence, it uses the algorithm topology of the knowledge module as well as past experiences. Monitoring the results of a specific aggregation enables the learning of functional aggregations and improves performance over time. Therefore, the cognitive module is an elementary module of the CAAI and the reason it is called a cognitive architecture. 


\subsection{Cognition}

The cognition module is a crucial part of the CAAI architecture, as it enables the system to learn over time. Subsequently, resulting knowledge can be transferred to other similar use cases (G-3). It is responsible for major tasks in the CAAI architecture, such as the algorithm selection, parameter tuning, and system management. To properly address these tasks, the following preconditions have to be fulfilled:

(i) Feature engineering is the task of selecting and extracting relevant features from sensor data after or during the pre-processing. Involving domain knowledge and years of experience from the engineers is considered a prerequisite and can significantly speed up process time and boost the quality of resulting models.

(ii) A declarative goal for the system has to be given, e.g., "minimize energy consumption". Furthermore, the goal needs to be reflected in the CPPS and the sensor data. A set of appropriate algorithms to address the specified goal has to be available.

(iii) Finally, relevant knowledge and business logic to solve the given task must be available.

As illustrated in Fig. 3, the cognition module works in two phases, initialization and operation.

\subsubsection{Phase 1: Initialization}

The cognitive module chooses one or more processing pipelines. Pipelines typically consist of pre-processing, modeling, and one or more model applications (such as classification, regression, or optimization). While some modules may require particular predecessors, e.g., a certain pre-processing, other module instances can be identical across different pipelines, and consequently, their results must be computed only once. The CAAI-BDP orchestrates the sequence of modules and manages their processing, which might be in parallel. The initialization phase utilizes automated selection tools such as AutoML or hyperheuristics. We implement Surrogate Model-Based Optimization (SMBO) to model the performance of algorithms and suggest new promising algorithm configurations by utilizing model-predictions $[5,44]$. Once pipelines are selected, the initialization step includes tuning the associated parameters of all included methods, to omit false configurations and wrong parameter settings. At the end of phase 1 , the cognition evaluates the candidate pipelines and chooses the best according to their performance.

\subsubsection{Phase 2: Operation}

The cognition is responsible for observing the processing pipeline in an online manner to detect drifts or performance decreases during the operation phase. These can occur if circumstances change over time, e.g., the quality of a material used in the production process. When such situations appear, the cognition performs a re-calibration of the processing pipeline, which includes a new selection or reconfiguration of the modules. This feature allows the system to adapt to new situations in the production process automatically. Moreover, the performance monitoring of the data structure itself and the performance of the chosen algorithms on the data enable the system to learn over time which methods are suitable for solving desired tasks.

\section{Implementation}

In this section, we introduce the considered use case, followed by implementation details about the CBDP. Furthermore, we present the process description of how CAAI behaves and introduce the results of our implementation.

\subsection{Use case}

We evaluate the CAAI through its implementation for the Versatile Production System (VPS), which is located in the SmartFactoryOWL. The VPS is a modular production system, which processes corn to produce popcorn, which is used as packaging material. Typically, there are four VPS

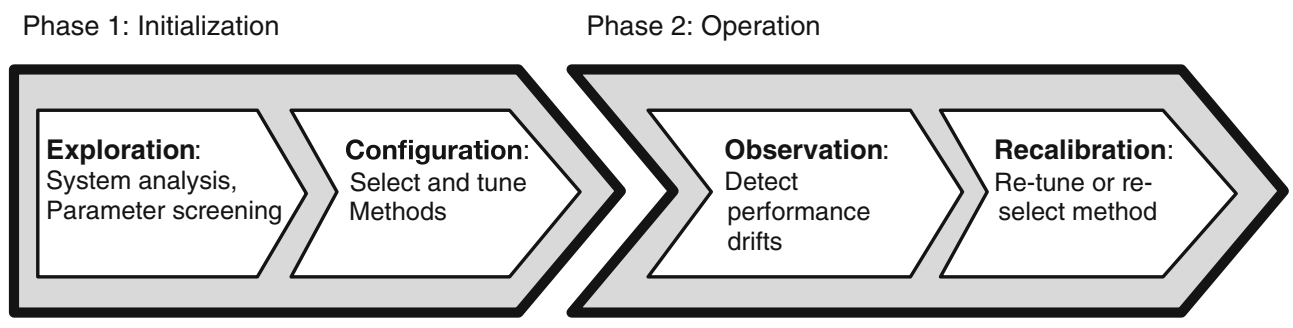

Fig. 3 Two working phases of the cognition module: Phase 1 initializes the search for the best fitting pipeline utilizing a space-filling design like Latin Hypercube Sampling. During phase 2 the process is observed with respect to performance drifts. A re-calibration of the methods can be performed on demand 
units, namely delivery, storage, dosing, and production. Due to its modularity, the first three units can be exchanged or removed easily. Depending on the current orders, different configurations are used. The need for different configurations rises, e.g., if the dosing unit should produce a small and exact amount of popcorn. However, if larger amounts are requested, it is more efficient to renounce the dosing unit because it is slow and generates operation costs. Efficiently operating the VPS is a challenge because many parameters influence the result, e.g., the moisture of the corn, the rate of corn that does not pop, or the amount of corn within the reactor. Since not all parameters can be measured inline, data-driven optimization is a promising method to increase efficiency. Therefore, the CAAI architecture perfectly matches the requirements of the VPS use case. As a basis, a reliable, easy to set up, and scalable environment for the AI is needed, which refers to method (M-1). Since the configuration is regularly changing, the AI components have to be modular (M-2), re-useable, and extendable.

Configurations of the VPS, the use case, and the VPS units might change over time. Hence, there is the need for cognition (M-3). However, improvements should be directly applied to the VPS to reach the best performance, where automatic decisions (M-4) are needed.

In this use case, all VPS units are used, and small boxes of popcorn are produced. In each batch, one box of popcorn has to be filled. The overage of popcorn produced in one batch, or not fully filled boxes cannot be used, so it is waste. Optimizing the amount of corn in the reactor, as provided by the dosing unit, is the goal. The optimum is a trade-off between three minimization functions: the energy consumption $\left(f_{1}\right)$, the processing time $\left(f_{2}\right)$, and the amount of corn needed for a small box $\left(f_{3}\right)$. These functions are conflicting to some degree. The result of the optimization is a parameter value for the dosing unit. The parameter $x$ controls the runtime of the conveyer and, therefore, indirectly influences the amount of corn processed. As the given optimization problem can be regarded as relatively simple, we will apply a single objective optimization algorithm and compute a weighted sum of the objectives. This results in the following optimization problem:

$\min \sum_{i=1}^{3} w_{i} f_{i}(x) ; \quad$ w.r.t $w_{i}>0$ and $\sum_{i=1}^{3} w_{i}=1$

The scalar weights of the corresponding objectives, $w_{i}$, are chosen based on the user's preferences. As a default, equal weights are used. The minimum of Eq. 1 is a Paretooptimal solution [31]. The problem will be optimized by SMBO [19]. SMBO utilizes a data-driven surrogate model to create an approximation of the real VPS production process. The model construction requires sampled data for a set of $n$ values of $x$, which should ideally depict a representative set of all possible settings, i.e., in a spacefilling manner. In this case, the set is generated by evaluating an equidistantly spaced design in the complete parameter range of $x$. The cognition will evaluate different surrogate models: random forest [7] and Kriging [23]. Kriging is especially suitable for modeling continuous data with few variables and comes with an uncertainty measurement. At the same time, random forest is also able to model discrete parameters and computes very fast. Recent examples of Kriging and random forest applications in CPPS scenarios can be found in [20,48].

With these two modeling algorithms, a broad range of systems can be covered. Furthermore, these surrogate models may differ in their hyperparameters, which results in a large number of possible configurations. The cognition decides which model and parameterization fit best to approximate the process data and perform optimization based on performance evaluation of the whole optimization cycle. The surrogate will then be optimized to identify the next candidate solution to be evaluated on the VPS by applying a local search algorithm. Figure 4 shows the optimization cycle of SMBO.

If a new parameter has been identified the business logic defines when an adaption should be performed and verifies boundaries, such as limitations for parameters to protect the VPS. Finally, the adaption module adapts the VPS, i.e., changes parameters to achieve better performance.

\subsection{CAAI-Big Data platform}

The CAAI-BDP is a distributed system that can be hosted on a single machine, an on-premise cluster, or a cloud provider. Our stated architectural goals (G1-G4) are supported by the CAAI-BDP through the implementation technologies, which are presented below. Transferring incoming/outgoing

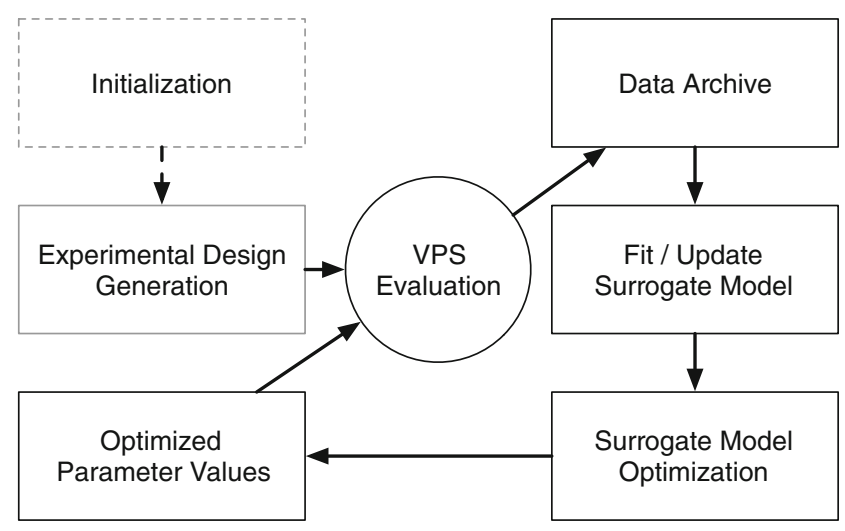

Fig. 4 The SMBO optimization cycle starts in the upper left with the initialization and design generation. Then the loop is processed with the evaluation of the design, the model computation and optimization to retrieve the next candidate solution to be evaluated in the VPS 
data, orchestrating the data processing tasks, assuring the persistence of results, and managing the communication between modules are the resulting tasks of the CAAI-BDP. The following concepts and technologies (T) are used to accomplish the tasks.

(T1) Container Virtualization: All components of the system exist as virtualized containers on the CAAIBDP. Isolating the module requirements from the general environment ensures that all requirements for a specific module are met and do not interfere with other modules on the same platform, similar to virtual machines. In contrast to virtual machines, a container uses the host operating system, and containers share binaries and libraries, resulting in less overhead. Containers are consistent and immutable, which ensures compatibility across systems. A central container registry stores the container images and keeps track of changes via versioning. Docker is used as a container engine for the implementation of this use-case [33]. Images consist of all the necessary code instructions to install the requirements and create a specific environment to execute the desired algorithm or software. Generally speaking, a validated, running image guarantees to work the same on every computer, server, or cloud environment.

(T2) Orchestration: The CAAI-BDP manages the necessary infrastructure and orchestrates virtualized components to compose a system consisting of microservices that perform a specific task. Orchestration frameworks handle deployments, configuration, updating, and removing of the virtualized software components. A text file declaratively composes a system and lists the different services. Orchestration is done by Kubernetes, which can utilize the Docker container engine [17]. The cognition module uses the orchestration to instantiate pipelines with selected algorithms and evaluate the results.

(T3) Microservices: All modules are developed as microservices to compose the software system for a specific use case from smaller self-sufficient parts. Each module includes standardized communication functionality to publish and subscribe to relevant topics on the bus system [46]. The resulting system is modular, language-agnostic, and utilizes welldefined interfaces. According to microservice bestpractices, each microservice can store internal data in its local storage.

(T4) Messaging: The different bus systems managed by the CAAI-BDP transfer data via messaging. Messaging allows asynchronous communication between modules and enables parallelization and processing data several times for different purposes via topics and consumer groups. Adding more instances to the same consumer group would result in a distributed processing of incoming messages, which is useful if a task is very time-consuming or response time is restricted. We chose Kafka [32] as a reliable message system for our platform.

(T5) Schema Management: A schema stores the metadata of the data, with all the available fields and datatypes [11]. When a module publishes to the bus system, the serializer applies the schema and encodes the message or filters out non-conforming messages. A consumer that subscribes to a topic on the bus has access to the same schema and can verify the integrity before encoding the incoming message. Therefore, clear communication via the bus system is ensured, and additional modules can be integrated easily. A central schema registry distributes and versions the schemas which allow regulated data evolution.

The combination of technologies (T1-T5) supports the overall goals and the methods to reach those, namely providing a reliable infrastructure (M-1) for modular development (M-2), e.g., re-using existing modules or extending the system with additional algorithms. The big data platform enables the cognition to run and evaluate additional experiments through the automatic creation of processing pipelines (M-3) and an automatic adaption of the CPPS (M-4) if a feasible and beneficial solution was found. The interaction of the five technologies is illustrated in Fig. 5.

\subsection{Process description}

Our architecture uses to select different algorithms and evaluate their results. The workflow of the architecture is depicted in Fig. 6. Its modularity (M-2) enables a convenient implementation of a SMBO algorithm as the functionality of single modules can be re-used. The cognition receives necessary information from the knowledge module and starts the workflow consisting of the following nine steps:

1. The cognition initializes candidate pipelines for parallel processing by varying model types and parameters. The knowledge module provides the required information about feasible algorithms and boundary constraints. Suitable models for this use case in our algorithm collection are either Kriging or random forest.

2. The protocol translation module transfers the data from the Open Platform Communications Unified Architecture (OPC UA) server on the CPPS to the data bus on the CAAI-CBDP.

3. The pre-processing module cleans the raw data. As the data quality is good, the pre-processing in this use case 
Fig. 5 Illustration of the five technologies that compose the CAAI-BDP. Images of the different modules are stored in the container registry (T1). The orchestration (T2) uses these images to instantiate the message broker (T4) as well as the schema registry (T5), to enable standardized communication between modules. Following that, the cognition instructs the orchestration, which modules compose a data processing pipeline (T3)

is reduced to data normalization. Figure 7 shows the pre-processing module publishing messages to the data bus for further (parallel) processing.

4. The Kriging and random forest model learning components fit or update their parameters and send the results to the analytics bus.

5. The module model application + optimization implements the sequential step of the SMBO algorithm: it searches the previously fitted model until an optimal solution is found or the maximum number of iterations is reached. The module transfers the result to the analytics bus.

6. The cognition decides using the model accuracy and predicted optimum, which pipeline will be chosen.

7. The business logic module verifies if the solution violates any of the constraints, e.g., too much corn in the reactor, and communicates the appropriate adaption back to the analytics bus.

8. The adaption module translates the adjustments for the specific CPPS and sends the instructions from the CAAI-CBDP to the CPPS.

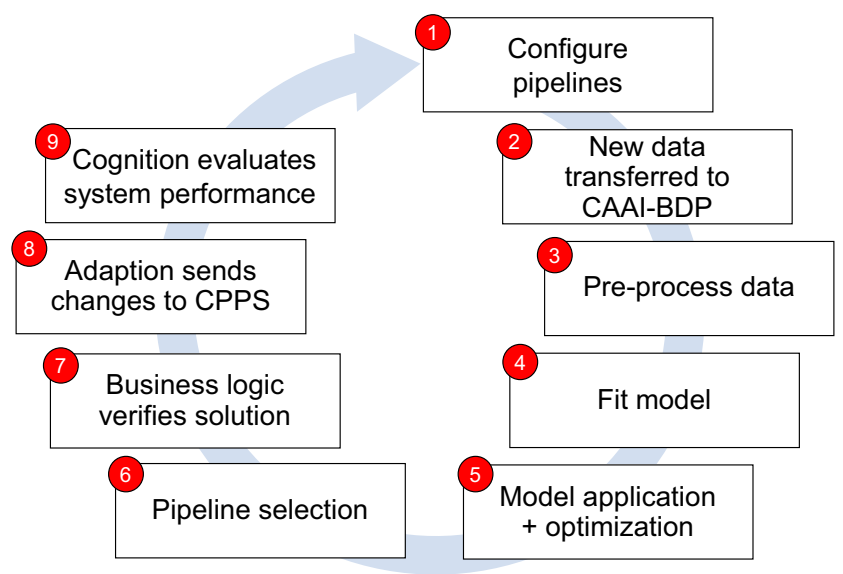

Fig. 6 The workflow represents nine steps that are continuously performed to adapt the pipelines and increase their performance over time
9. The cognition module analyses the system performance, as achieved with the resulting pipeline configuration from step 6 . In the following steps, the impact of changes is verified through information provided by the monitoring module.

The resulting implementation of the CAAI for the given use case, including all applied modules and the described workflow, is illustrated in Fig. 8.

\subsection{Results}

Data from the real-world VPS was acquired to evaluate the modeling and optimization. This data consists of 36 production cycles with 12 different settings for the runtime of the conveyor. Based on this data, we trained a model that reflects the real behavior of the VPS and utilize it for further experiments. The three different objectives, i.e., the energy consumption, the processing time, and the amount of corn needed (see Section 3.1 for more details), were aggregated by taking the sum of the single objectives multiplied with equal weights of $1 / 3$.

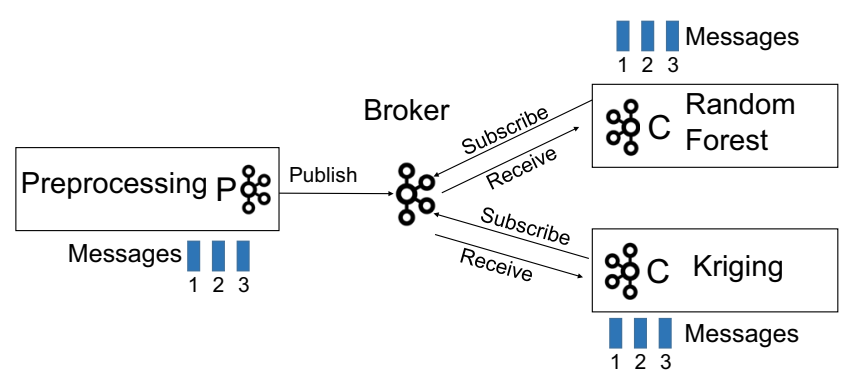

Fig. 7 Parallel processing of messages through different algorithms. The cognition module instantiated two pipelines with candidate algorithms and assigned them into different consumer groups. As two consumer groups are subscribed to this topic, both groups receive all new messages and various algorithms can be trained independently. More instances can be added to the same consumer group, which results in a distributed processing of incoming messages 
Fig. 8 The resulting CAAI architecture for the VPS use case. The numbers indicate the sequence of the workflow, where some steps can be computed in parallel, i.e., the two different surrogate models

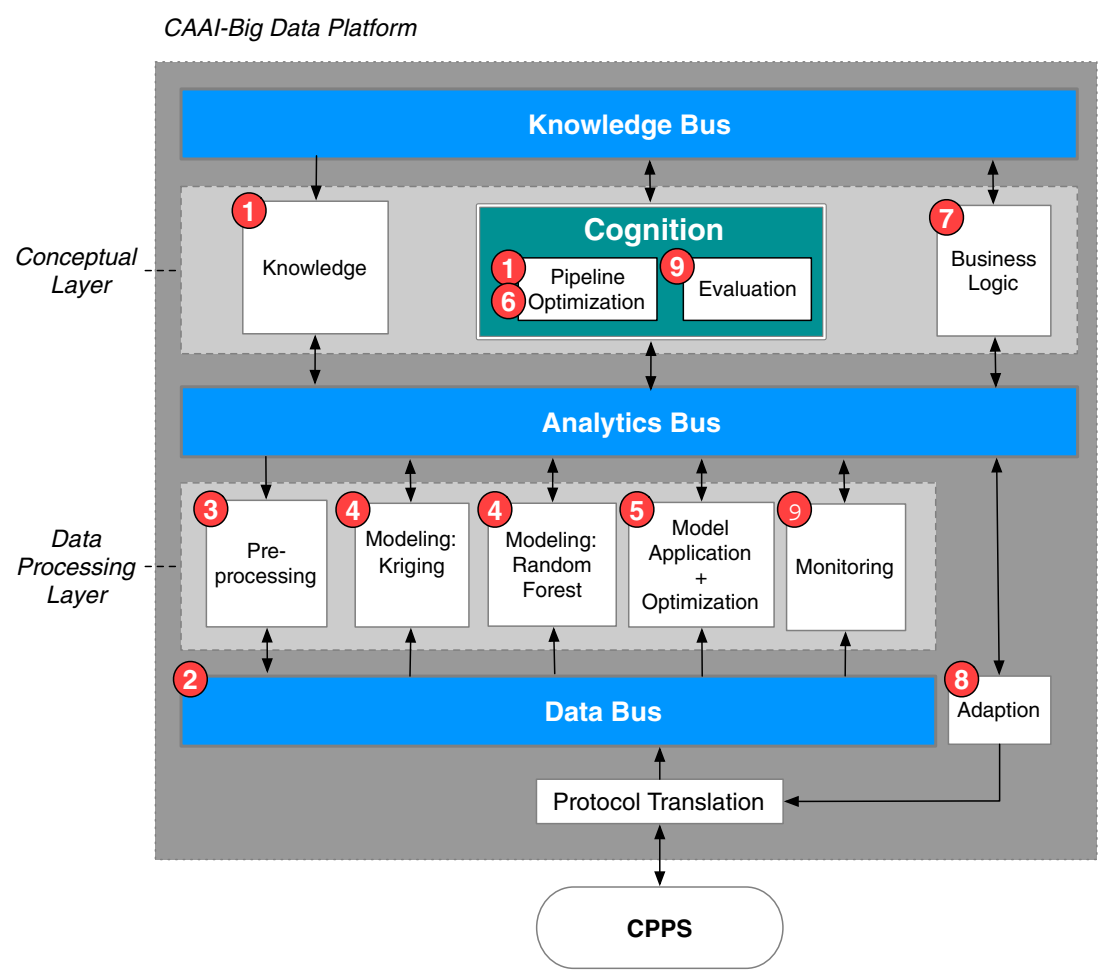

A MacBook Pro with a quad-core Intel Core i7 CPU at $3.1 \mathrm{GHz}$ and $16 \mathrm{~GB}$ DDR3 RAM computed the results described in this Section. The statistical software $\mathrm{R}$ version 3.4.3 was used to evaluate the algorithms [38]. Kriging and random forest employ the software packages SPOT (2.0.5) [4] and caret (6.0-84) [24]. In the initial phase, the algorithms used five equidistant data points to build their initial models. Consequently, the results in the figures presented in this section start at production cycle number five. The aggregated results use the median values of ten repetitions, with 20 production cycles in each repetition. Figure 9 plots the CPU consumption in seconds against the VPS production cycles.

For both methods, Kriging and random forest, an increasing trend can be observed. However, the computation time of Kriging shows a larger slope, compared with random forest. Both algorithms behave as expected, stemming from the internal data representation and processing. The same holds for memory consumption, as depicted in Fig. 10. At the early stage, the random forest algorithm uses more RAM than Kriging. After about 15 iterations the situation changes as Kriging started to acquire more memory than random forest. While the required memory grows further for both algorithms, Kriging also shows the steeper slope.

Comparing the prediction accuracy of the models at their best-predicted points with the real objective function value, as shown in Fig. 11, Kriging shows a nearly constant accurate performance, while random forest shows a larger variance and starts to get comparably accurate predictions in the last production cycles.

The reached values of the objective function are depicted in Fig. 12. It shows that in the beginning, Kriging outperforms random forest, while later after about 12 cycles, random forests perform comparably to Kriging.

Results from our study indicate the following, valuable findings:

(i) It is worth using more than one algorithm: taking only the best performing (i.e., Kriging) can lead to future problems due to limited computation resources and time.

(ii) Random forest needs more data to improve compared with Kriging and starts to be a good competitor after about 15 cycles.

(iii) The prediction error is a useful measure to detect performance drifts and switch to other pipelines if needed.

(iv) Altogether, it is beneficial to switch algorithms after a certain number of production cycles, when regarding all performance metrics together.

(v) A forgetting mechanism is necessary to implement SMBO efficiently for long-term usage in CPPS scenarios due to physically limited computation resources. This mechanism could be a fixed or adaptive size of the model, e.g., the sliding window approach applied in time series computation, and a method to choose which data to remove from the model. 
Fig. 9 This plot shows the consumed CPU time in seconds over the production cycle, which is equal to the number of data points used. The results are aggregated over 10 repetitions using median values

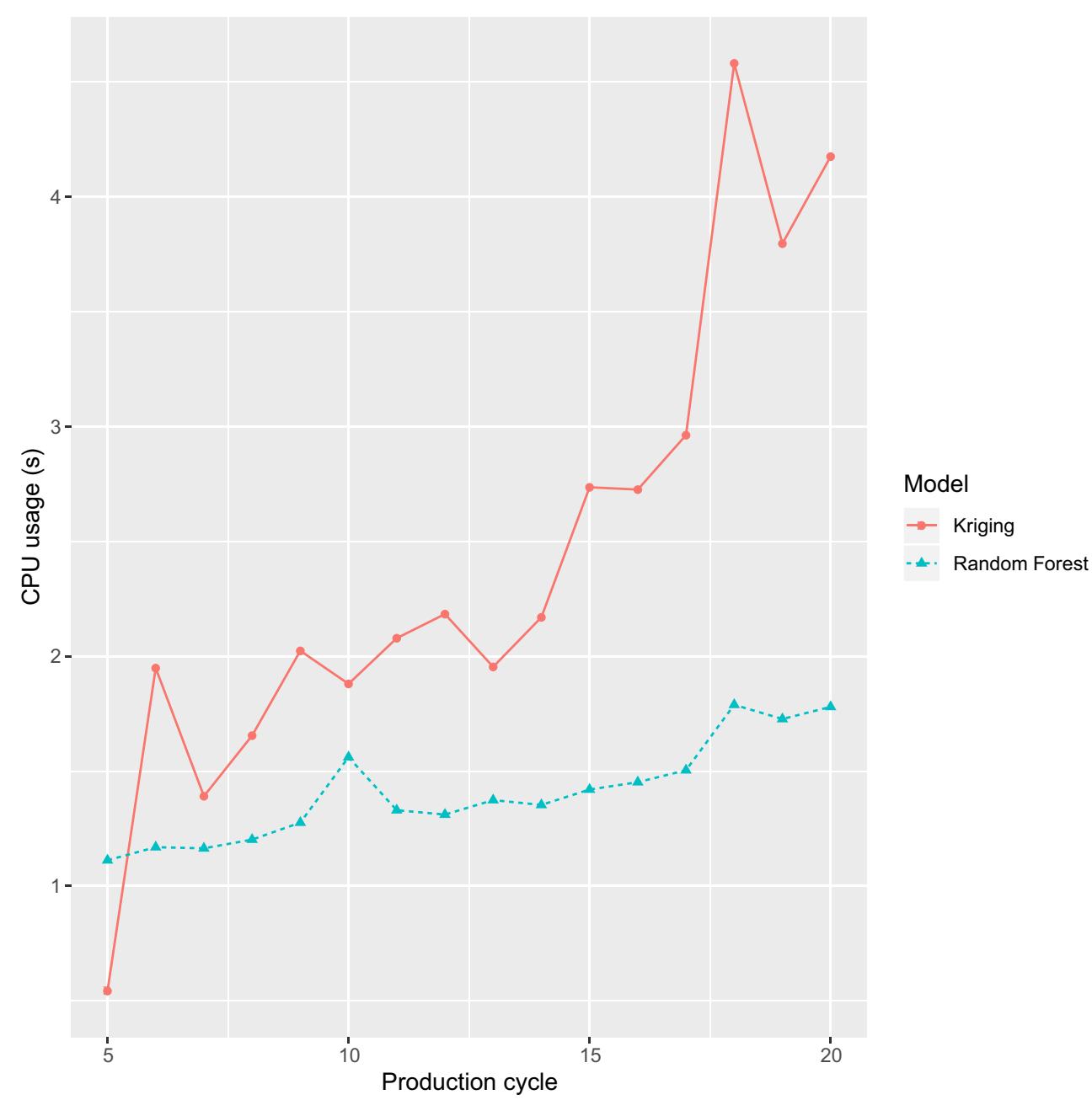

Additionally, the processed material can change its behavior over time. Storing the corn over an extended period eventually requires more corn to fill the cups with the desired amount. Therefore, it is crucial to adapt the system during runtime by changing the computation pipelines, recomputing models, and adjusting model sizes.

The cognition module uses the aggregated information shown in Figs. 9, 10, 11, and 12 to select the best algorithm for the next production cycles. This also demonstrates the relative degree of information density on the analytics bus compared to the data bus (see Table 1). The cognition module does not use the raw data, which comprises several hundred rows of data for each production cycle.

A prototypic implementation of this use case is hosted publicly on GitHub ${ }^{2}$. The related tutorial shows how to start the big data platform and the processing pipeline as a group of Docker containers. The program flow and the communication of the individual modules can then be followed in the console.

\footnotetext{
$\overline{{ }^{2} \text { https://github.com/janstrohschein/KOARCH/tree/master/Use_Cases/ }}$ VPS_Popcorn_Production/Docker
}

\section{Conclusion and outlook}

In this paper, we defined goals (G1-G4) to be reached by a cognitive architecture to improve or maintain the efficiency of a CPPS. Each goal is addressed by a particular method (M1-M4), which can be implemented by several technologies, e.g., (T1)-(T4) or solutions. Figure 13 details the coherence of these goals, methods, and solutions which results in our cognitive architecture CAAI, which was presented, and further evaluated on a real-world problem in this work. Different manifestations of this architecture are possible, with one implementation being exemplified for the VPS use case evaluation. The key feature is the cognition module that configures, instantiates and evolves process pipelines over time to solve the problem, i.e., to reach the addressed goal.

Besides the main contribution of this paper, the proposed CAAI, the major results (R) can be summarized as follows:

(R-1) The central aim, to efficiently optimize the performance of a CPPS, can not be reached by addressing 
Fig. 10 This figure depicts the memory (MB) consumption of the modeling algorithms for different production cycles. The number of the production cycle is equal to the number of data points used in this cycle. The results are aggregated over 10 repetitions using median values

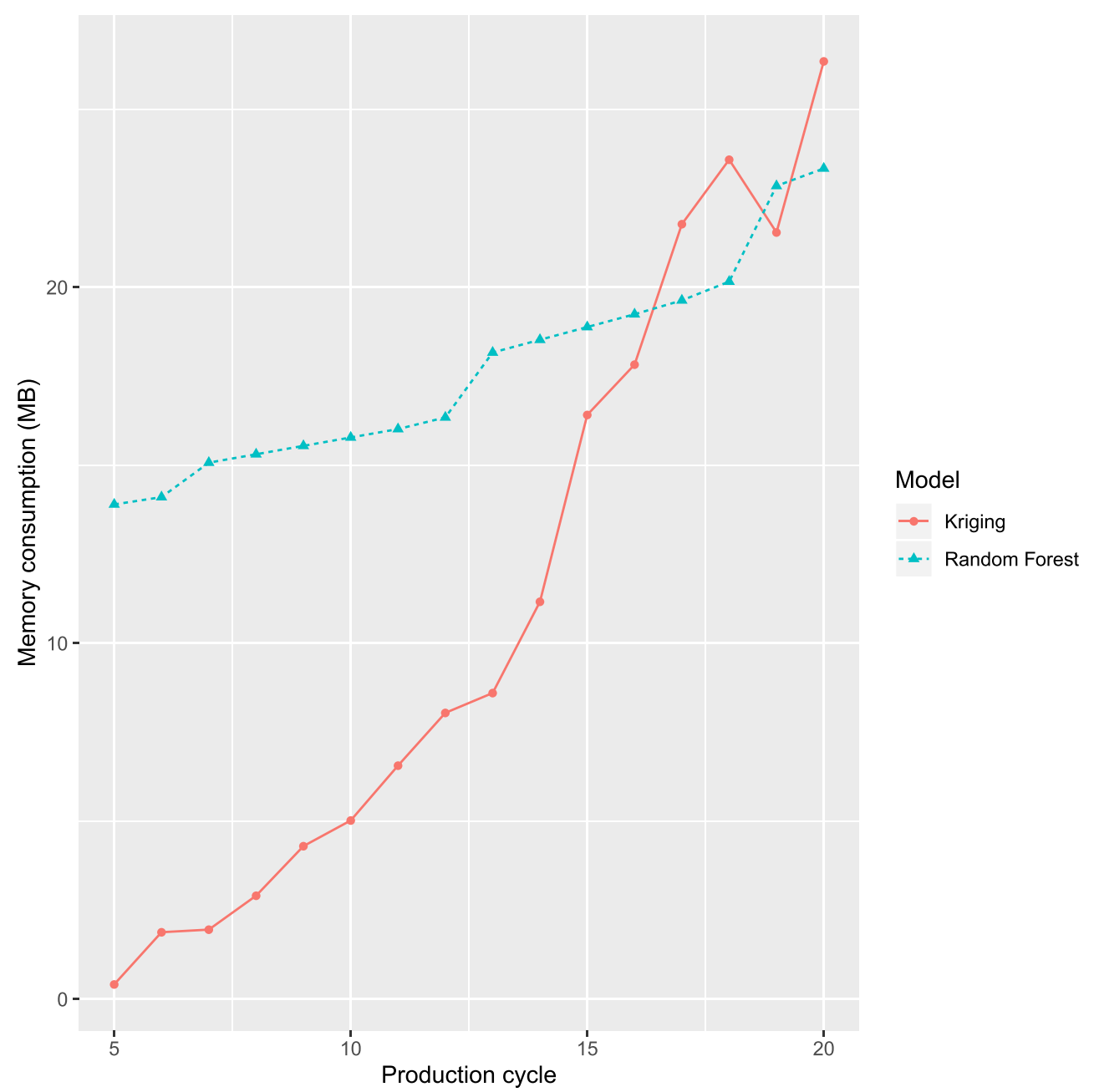

single goals or implementing single methods individually (see Section 1). The main implication is that the individual goals support each other to a high degree. For example, a flexible and modular system is required by the cognition component to allow generalization of the system and apply to different use cases.

(R-2) The defined goals and methods represent the reference character of the proposed architecture and will maintain valid over a long time. However, the solutions and chosen technologies or concepts for implementation may be subject to change in the future. For example, Docker is a solid choice to fulfill the requirements of CPPS scenarios, to process data in near real-time (see Section 3). As technologies evolve, this solution may be replaced by a more modern one in the future but can be regarded as a state-of-the-art virtualization method nowadays.

(R-3) The performance of an algorithm for a given use case and data may change over time, in both directions (worsening and improving) (see Section 3.4).
Therefore, an offline benchmark and selection, which chooses one particular algorithm as the best performing, might be misleading. Moreover, a particular algorithm's processing time can significantly increase due to growing data volume, e.g., through additional sensors in the CPPS. Hence, changing the processing pipeline to another algorithm can be beneficial to reduce computational resources costs. The cognitive module needs sufficient degrees of freedom and a somewhat broad portfolio of algorithms to realize such an online selection and establish an efficient system.

(R-4) Overall, the CAAI is specific enough to support concrete implementation in the form of the CAAIBDP. The resulting system was able to optimize a simple I4.0 use case by configuring, instantiating, and evaluating several processing pipelines.

The proposed facilitation reference architecture aims to support the user with an efficient implementation for a specific use case. Consequently, several disciplines, i.e., data, knowledge, and algorithms, are combined and 
Fig. 11 Prediction error plotted against the number of production cycles. The prediction error is the absolute difference between the model prediction of the best found point and the true objective function value

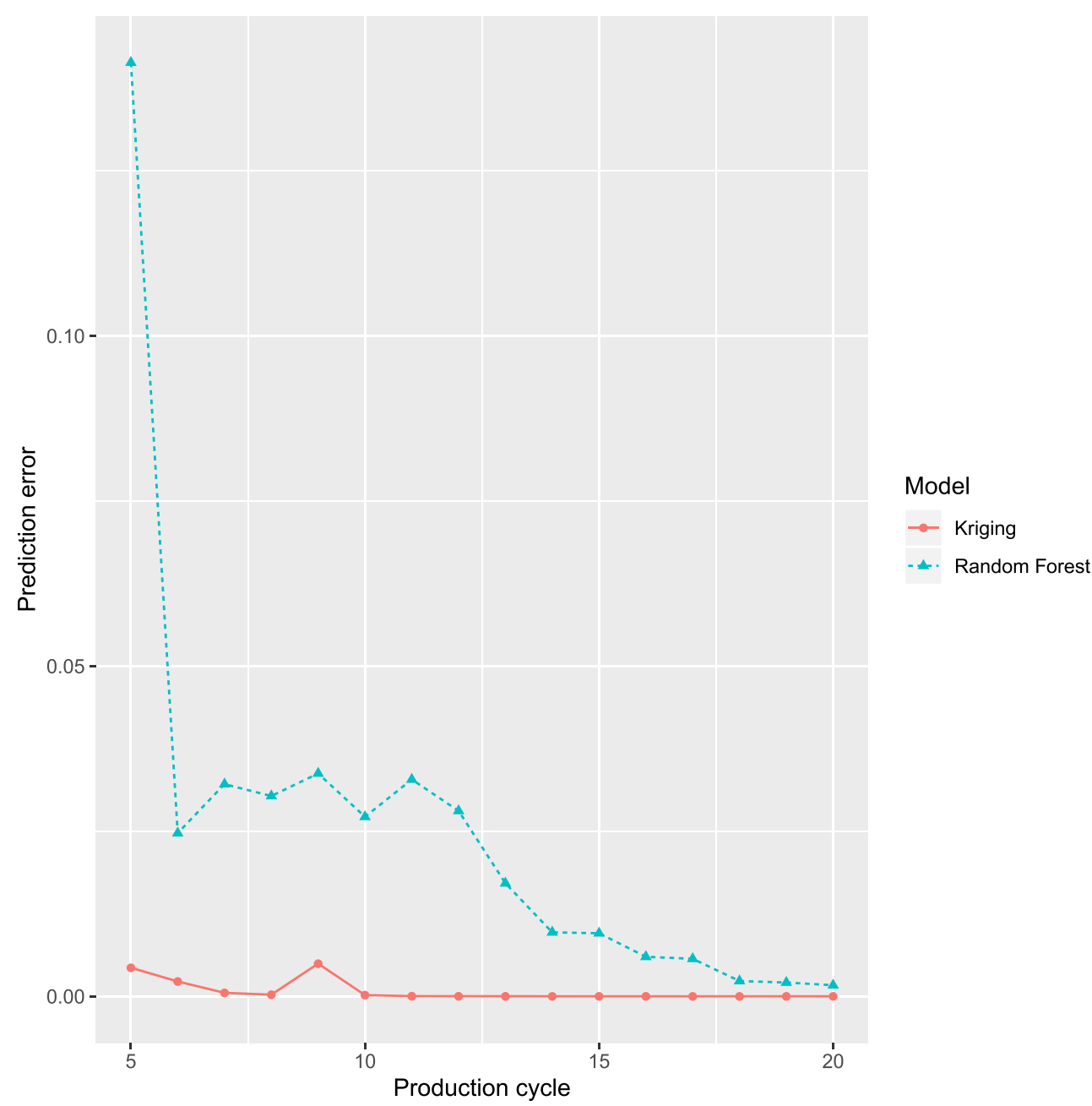

interconnected to enable artificial intelligence for I4.0 scenarios. State of the art does not provide solutions for all problems in these disciplines, especially when different use cases are regarded. Therefore, the architecture is developed in a flexible and extendable manner, such that future solutions can be easily integrated by implementing and adding new modules.

The cognition needs pre-classified algorithms to sample from and learn, which algorithm class is superior to others, regarding the current use case. A suitable algorithm taxonomy for optimizers based on the implemented features of the algorithms can be found in [3]. To evaluate the performance of selected algorithms, the estimated runtime of an algorithm to reach a desired optimization target value is a valid choice [22]. We suggest aggregating this pure performance metric with the resources consumption measurement per algorithm. The resource consumption is very interesting, especially for applications in small and medium-sized companies, where no high-performance cluster or, probably expensive, cloud solutions may be available.
Regarding the management of data and representation of knowledge, $\mathrm{Li}$ et al. provide an overview of corresponding methods and applications in industrial environments [28]. Please note that the method should be selected carefully with respect to the compatibility of the knowledge representation in the I4.0 environment. Nevertheless, the selection of suitable technology is out of the scope of this paper. To reference the needed knowledge at runtime, e.g., to select suitable algorithms according to the algorithms family and the according control parameters, implementing the knowledge module as an Application Programming Interface (API) allows the modification of the knowledgebase properly either manually by the user or automatically by the cognition module. The OpenAPI initiative defines a language-agnostic interface description for API building blocks [43]. When properly defined, the microservices can interact with the API building block, e.g., the knowledge, with minimal implementation logic.

Data integration from CPPS can be dynamically performed by OPC UA. OPC UA is a platform-independent standard that aims to exchange information between 
Fig. 12 Objective function value plotted against the number of production cycles

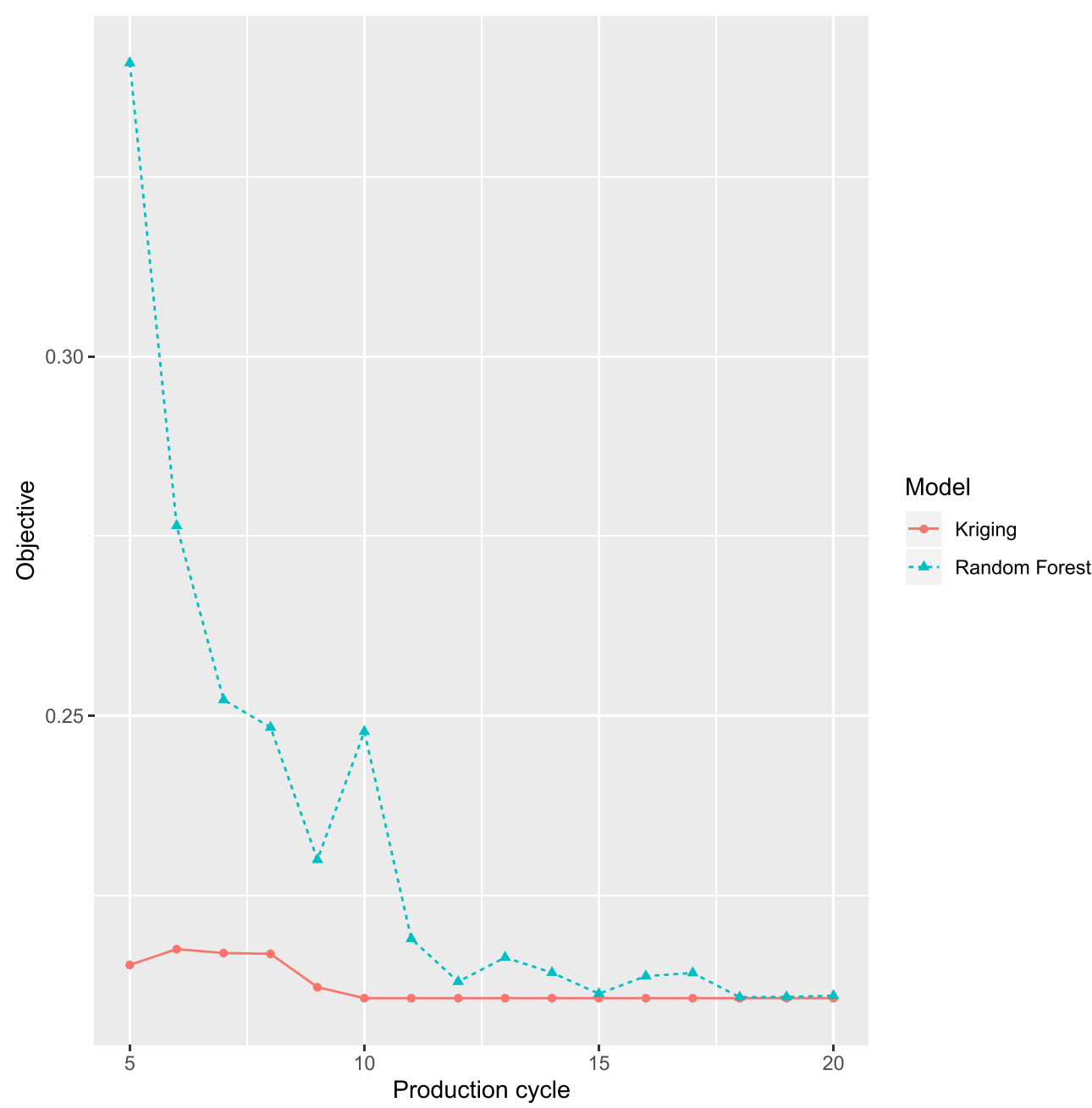

automation systems [40]. It is located on the upper levels of the ISO/OSI Model and thus does not depend on a defined communication protocol [9]. OPC UA provides high-level functionalities such as a discovery service that allows to discover, connect to, and access servers in the network automatically [37]. Signals can be described using an information model. Consequently, if the CPPS supports a technology such as OPC UA, data integration can be performed automatically.

Building a message-driven big data platform increases software interoperability as all messages regarding a particular topic need to follow the defined schema. Standardized interfaces simplify the extension via additional modules. Using containerized environments enables a languageagnostic development, e.g., it is possible to use algorithm implementations in R and Python side-by-side. Thus, it is possible to use the best available implementation for a given algorithm.

From our point of view, the outcomes of the paper at hand cover three important disciplines in the field of AI-applications and the related methods: (i) Infrastructure: Must be reliable and flexible to fulfill response time requirements in industry scenarios (M1 and $\mathrm{M}-2)$.

(ii) Learning: Suitable algorithm portfolio and topology needed to address several declarative goals and resource limitations (M-3).

(iii) Data curation: Combines data pre-processing and domain knowledge to create metadata to support further algorithmic processing (M-3 and M-4).

To discuss open issues, automatic feature extraction or explainable AI are important topics to be mentioned. However, the flexibility of the architecture enables easy integration of novel technologies. The development of such solutions is out of the scope of this paper, as we focus on architectural concepts and the framework to integrate such technologies.

This paper's work reveals some additional questions, leading to logical next steps and follow-up research tasks: The effort to solve a different use case by implementing and adjusting the CAAI, which should be minimized, has 


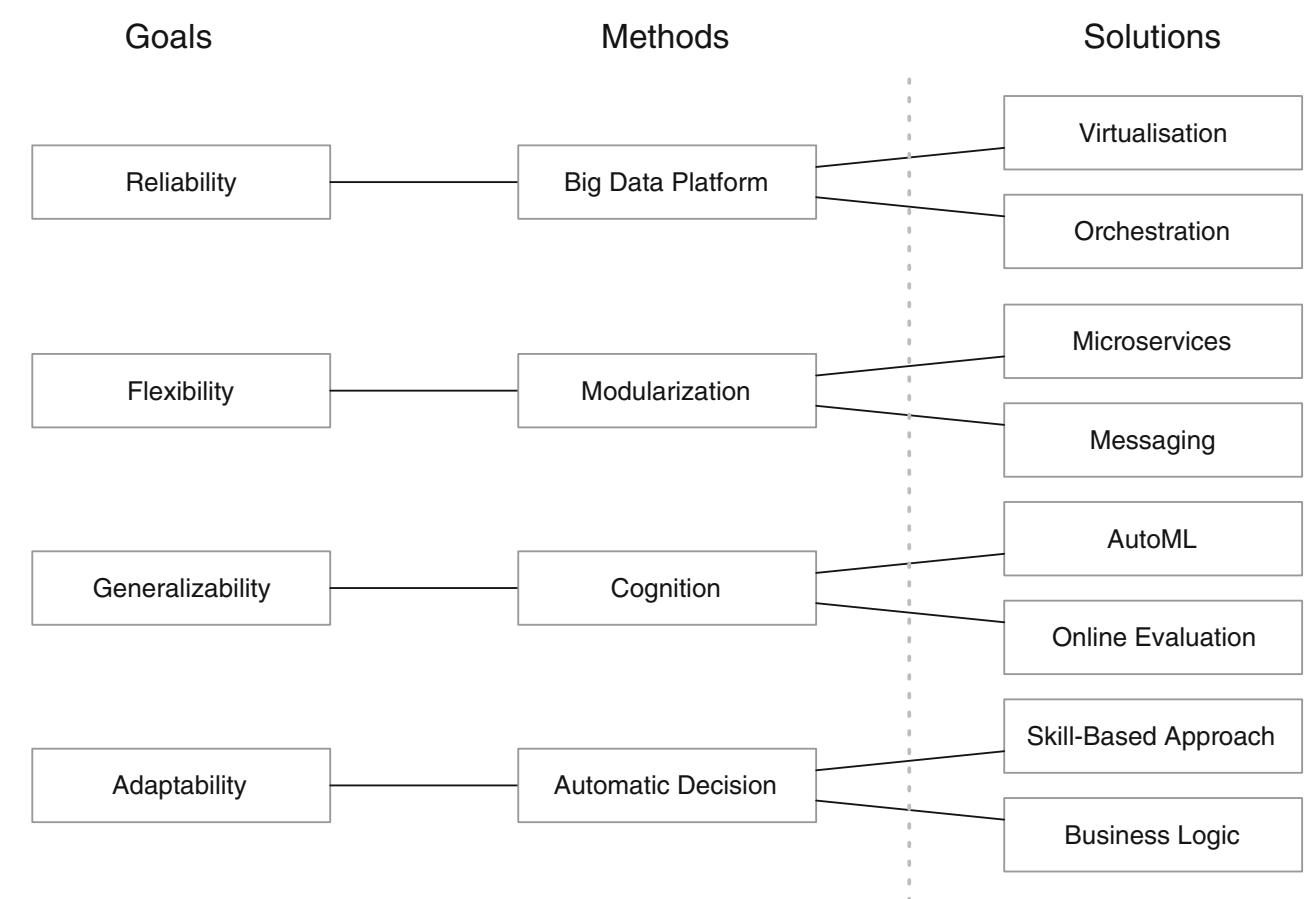

Fig. 13 Overview of goals, methods and solutions. The goals and methods on the left side represent the parts with a larger reference character of the architecture, while the solutions on the right side rather represent the current state of the art in the respective disciplines

to be analyzed. This would directly take up results (R2 ), regarding the reference character of the CAAI, and (R-3), regarding the algorithm portfolio. Additionally, e.g., configuration for the orchestration, time requirements, or data pre-processing, may change as well.

The implementation of pipeline creation and evaluation is adequate for the presented, in terms of the optimization problem rather straightforward, use case. However, further efforts are required to build a truly intelligent system that can solve harder use cases through learning over time and re-calibration in an online manner (R-3). Automatically adapting the CPPS was not yet considered for the regarded use case, as we focus in particular on the CAAI-BDP and the cognitive module. Therefore, our future work will include a representation of the necessary knowledge to safely change the configuration and operation of the CPPS while trying to increase its efficiency.

To give an idea of a possible long-term perspective, the degree of cognition addressed by our architecture can be the topic of future research. We restricted the scope of the cognition to typical I4.0 use cases, see Definition 1. An extension of the cognitive capabilities to detect restrictions (e.g., introduced by the provided algorithms or the limited available resources) independently and learn how to deal with them autonomously would be the next step towards a self-aware system.

Funding Open Access funding enabled and organized by Projekt DEAL. The work was supported by the German Federal Ministry of Education and Research (BMBF) under the project "KOARCH" (funding code: 13FH007IA6 and 13FH007IB6).

\section{Compliance with ethical standards}

Conflict of interest The authors declare that they have no conflict of interest.

Open Access This article is licensed under a Creative Commons Attribution 4.0 International License, which permits use, sharing, adaptation, distribution and reproduction in any medium or format, as long as you give appropriate credit to the original author(s) and the source, provide a link to the Creative Commons licence, and indicate if changes were made. The images or other third party material in this article are included in the article's Creative Commons licence, unless indicated otherwise in a credit line to the material. If material is not included in the article's Creative Commons licence and your intended use is not permitted by statutory regulation or exceeds the permitted use, you will need to obtain permission directly from the copyright holder. To view a copy of this licence, visit http:// creativecommonshorg/licenses/by/4.0/.

\section{References}

1. Adolphs $\mathrm{P}$ et al (2015) Reference Architecture Model Industrie 4.0 (RAMI4.0). Tech. rep., VDI. https://www. zvei.org/fileadmin/user_upload/Themen/Industrie_4.0/ Das_Referenzarchitekturmodell_RAMI_4.0_und_die_Industrie_4. 0-Komponente/pdf/5305_Publikation_GMA_Status_Report_ ZVEI_Reference_Architecture_Model.pdf. Accessed: 2020-02-18

2. Anderson JR (1996) ACT: A simple theory of complex cognition. Am Psychol 51(4):355-365. https://doi.org/10.1037/0003-066X. 51.4.355

3. Bartz-Beielstein T, Doerr C, Bossek J, Chandrasekaran S, Eftimov T, Fischbach A, Kerschke P, Lopez-Ibanez M, Malan KM, Moore JH, Naujoks B, Orzechowski P, Volz V, Wagner M, Weise T 
(2020) Benchmarking in optimization: best practice and open issues. arXiv:2007.03488

4. Bartz-Beielstein T, Gentile L, Zaefferer M (2017) In a nutshell: sequential parameter optimization. arXiv: 1712.04076

5. Bartz-Beielstein T, Zaefferer M (2017) Model-based methods for continuous and discrete global optimization. Appl Soft Comput 55:154-167. https://doi.org/10.1016/j.asoc.2017.01.039

6. Bauer K, Diemer J, Hilger C, Löwen U, Michels JS (2017) Benefits of application scenario value-based service. Tech. rep., Federal Ministry for Economic Affairs and Energy (BMWi). https://www. plattform-i40.de/PI40/Redaktion/DE/Downloads/Publikation/ benefits-application-scenario.pdf?__blob=publicationFile \&v=7. Accessed: 2020-02-18

7. Breiman L (2001) Random forests. Mach Learn 45(1):5-32

8. Bunte A, Fischbach A, Strohschein J, Bartz-Beielstein T, Faeskorn-Woyke H, Niggemann O (2019) Evaluation of cognitive architectures for cyber-physical production systems. In: 24nd IEEE International Conference on Emerging Technologies and Factory Automation (ETFA), Zaragoza, Spain, pp 729-736. https://doi.org/10.1109/ETFA.2019.8869038

9. Bunte A, Niggemann O, Stein B (2018) Integrating owl ontologies for smart services into automationml and opc ua. In: 23Rd IEEE International Conference on Emerging Technologies and Factory Automation (ETFA), Turin, Italy, pp 1383-1390. https://doi.org/10.1109/ETFA.2018.8502593

10. Bunte A, Wunderlich P, Moriz N, Li P, Mankowski A, Rogalla A, Niggemann O (2019) Why symbolic ai is a key technology for self-adaption in the context of cpps. In: 24nd IEEE International Conference on Emerging Technologies and Factory Automation (ETFA), Zaragoza, Spain, pp 1701-1704. https://doi.org/10.1109/ETFA.2019.8869082

11. Confluent (2020) Schema management. https://docs.confluent.io/ current/schema-registry/index.html. Accessed: 2020-02-18

12. Drath R, Horch A (2014) Industrie 4.0: Hit or hype? [industry forum]. IEEE Ind Electron Mag 8(2):56-58. https://doi.org/10.1109/MIE.2014.2312079

13. European Factories of the Future Research Association (EFFRA) (2013) Factories of the Future: Multi-annual Roadmap for the Contractual PPP Under Horizon 2020. EDC collection. Publications Office of the European Union. https://doi.org/10.2777/29815. https://books.google.de/books? id=ZC0wngEACAAJ. Accessed: 2020-02-18

14. Feurer M, Klein A, Eggensperger K, Springenberg JT, Blum M, Hutter F (2015) Efficient and robust automated machine learning. In: Proceedings of the 28th International Conference on Neural Information Processing Systems - Volume 2. MIT Press, Cambridge, MA, USA. https://dl.acm.org/doi/10.5555/2969442. 2969547

15. Fusi N, Sheth R, Elibol M (2018) Probabilistic matrix factorization for automated machine learning. In: Proceedings of the 32nd International Conference on Neural Information Processing Systems, p 3352-3361. Curran Associates Inc., Red Hook, NY, USA. https://dl.acm.org/doi/10.5555/3327144.3327254

16. Gokalp MO, Kayabay K, Akyol MA, Eren PE, Koçyiğit A (2016) Big data for industry 4.0: a conceptual framework. In: 2016 International Conference on Computational Science and Computational Intelligence (CSCI), pp 431-434. https://doi.org/10.1109/CSCI.2016.0088

17. Hightower K, Burns B, Beda J (2017) Kubernetes: up and running dive into the future of infrastructure, 1st edn. O'Reilly Media, Inc

18. IBM Knowledge Center (2019) Main features and benefits of message queuing. https://www.ibm.com/support/knowledgecenter/ SSFKSJ_9.1.0/com.ibm.mq.pro.doc/q002630_.htm. Accessed: 2020-02-18
19. Jin Y (2005) A comprehensive survey of fitness approximation in evolutionary computation. Soft Comput 9(1):3-12. https://doi.org/10.1007/s00500-003-0328-5

20. Jung C, Zaefferer M, Bartz-Beielstein T, Rudolph G (2017) Metamodel-based optimization of hot rolling processes in the metal industry. Int J Adv Manuf Techn 90(1):421-435. https://doi.org/10.1007/s00170-016-9386-6

21. Kagermann H, Wahlster W, Helbig J (2013) Securing the future of german manufacturing industry - recommendations for implementing the strategic initiative INDUSTRIE 4.0. Tech. rep., acatech - National Academy of Science and Engineering, Berlin. https://en.acatech.de/wp-content/uploads/sites/6/2018/03/ Final_report_Industrie_4.0_accessible.pdf. Accessed: 2020-02-18

22. Kerschke P, Trautmann H (2017) Automated algorithm selection on continuous black-box problems by combining exploratory landscape analysis and machine learning. arXiv:1711.08921

23. Krige D (1951) A statistical approach to some basic mine valuation problems on the witwatersrand. J Chem Metall Min Soc South Afr 52(6):119-139

24. Kuhn M (2008) Building predictive models in $r$ using the caret package. J Stat Softw 28(5):1-26. https://doi.org/10.18637/ jss.v028.i05

25. Laird JE, Newell A, Rosenbloom PS (1987) Soar: an architecture for general intelligence. Artif Intell 33(1):1-64. https://doi.org/10.1016/0004-3702(87)90050-6

26. Lee J, Jin C, Bagheri B (2017) Cyber physical systems for predictive production systems. Prod Eng 11(2):155-165. https://doi.org/10.1007/s11740-017-0729-4

27. Li D, Fast-berglund $\AA$, Paulin D (2019) Current and future industry 4.0 capabilities for information and knowledge sharing. Int $\mathrm{J}$ Adv Manuf Tech 105(9):3951-3963. https://doi.org/10.1007/s00170-019-03942-5

28. Li X, Zhang S, Huang R, Huang B, Xu C, Zhang Y (2018) A survey of knowledge representation methods and applications in machining process planning. Int J Adv Manuf Tech 98:30413059. https://doi.org/10.1007/s00170-018-2433-8

29. Lin SW et al (2017) The industrial internet of things volume G1: reference architecture v1.80. Tech. rep., Industrial Internet Consortium. https://www.iiconsortium.org/IIC_PUB_G1_V1. 80_2017-01-31.pdf. Accessed: 2020-02-18

30. Malakuti S, Bock J, Weser M, Venet P, Zimmermann P, Wiegand M, Grothoff J, Wagner C, Bayha A (2018) Challenges in skill-based engineering of industrial automation systems*. In: 23Rd IEEE International Conference on Emerging Technologies and Factory Automation (ETFA), Turin, Italy, pp 67-74. https://doi.org/10.1109/ETFA.2018.8502635

31. Marler R, Arora J (2010) The weighted sum method for multiobjective optimization: new insights. Struct Multidiscip Optim 41:853-862. https://doi.org/10.1007/s00158-009-0460-7

32. Narkhede N, Shapira G, Palino T (2017) Kafka: the definitive guide real-time data and stream processing at scale, 1 st edn. O'Reilly Media, Inc

33. Negus C (2015) Docker containers, 2nd edn. Addison-Wesley Professional

34. Neisser U (2014) Cognitive psychology: Classic edition. Psychology Press Classic Editions

35. Olson RS, Urbanowicz RJ, Andrews PC, Lavender NA, Kidd LC, Moore JH, Squillero G, Burelli P (2016) Automating biomedical data science through tree-based pipeline optimization. In: Applications of evolutionary computation. Springer International Publishing, Cham, pp 123-137. https://doi.org/10.1007/978-3-319-31204-0_9

36. Plattform Industrie 4.0 (2019) Technology Scenario “Artificial Intelligence in Industrie 4.0'. Tech. rep., Federal Ministry 
for Economic Affairs and Energy (BMWi). https://www. plattform-i40.de/PI40/Redaktion/EN/Downloads/Publikation/ AI-in-Industrie4.0.pdf. Accessed: 2020-02-18

37. Profanter S, Dorofeev K, Zoitl A, Knoll A (2017) Opc ua for plug \& produce: automatic device discovery using ldsme. In: 22Nd IEEE International Conference on Emerging Technologies and Factory Automation (ETFA), Limassol, Cyprus, 1-8. https://doi.org/10.1109/ETFA.2017.8247569

38. R Core Team (2017) R: a language and environment for statistical computing. R Foundation for Statistical Computing, Vienna. https://www.R-project.org/. Accessed: 2020-02-18

39. Santos MY, Oliveira e Sá J, Costa C, Galvão J, Andrade C, Martinho B, Lima FV, Costa E (2017) A big data analytics architecture for industry 4.0. In: Rocha Á, Correia AM, Adeli $\mathrm{H}$, Reis LP, Costanzo S (eds) Recent advances in information systems and technologies. Springer International Publishing, Cham. https://doi.org/10.1007/978-3-319-56538-5_19

40. Schilberg D, Hoffmann M, Schmitz S, Meisen T (2017) Interoperability in smart automation of cyber physical systems. Springer International Publishing, Cham, pp 261-286. https://doi.org/10.1007/978-3-319-42559-7_10

41. Schröder C (2016) The challenges of Industry 4.0 for small and medium-sized enterprises. Friedrich-Ebert-Stiftung, Division for Economic and Social Policy, Bonn. http://library.fes.de/pdf-files/ wiso/12683.pdf. Accessed: 2020-02-18

42. Shannon CE (1948) A mathematical theory of communication. Bell Syst Tech J 27(3):379-423. https://doi.org/10.1002/j.15387305.1948.tb01338.x

43. The Linux Foundation (2020) The openapi specification. https:// www.openapis.org. Accessed: 2020-08-18
44. Thomas J, Coors S, Bischl B (2018) Automatic gradient boosting. arXiv: 1807.03873

45. Thornton C, Hutter F, Hoos HH, Leyton-Brown K (2013) Autoweka: Combined selection and hyperparameter optimization of classification algorithms. In: Proceedings of the 19th ACM SIGKDD International Conference on Knowledge Discovery and Data Mining. Association for Computing Machinery, New York, pp 847-855. https://doi.org/10.1145/2487575.2487629

46. Watts K (2015) Microservices architecture: deep exploration of microservices. Create space independent publishing platform, north charleston, SC USA

47. Weskamp JN, Poudel BK, Al-Gumaei K, Pethig F (2019) Offene big-data-plattform für industrie 4.0. atp Magazin 61(3):96105. https://doi.org/10.17560/atp.v61i3.2377. http://ojs.di-verlag. de/index.php/atp_edition/article/view/2377

48. Xing B, Xiao Y, Qin QH, Cui H (2018) Quality assessment of resistance spot welding process based on dynamic resistance signal and random forest based. Int J Adv Manuf Tech 94(1):327339. https://doi.org/10.1007/s00170-017-0889-6

49. Zimmermann P, Axmann E, Brandenbourger B, Dorofeev K, Mankowski A, Zanini P (2019) Skill-based engineering and control on field-device-level with opc ua. In: 24Th IEEE International Conference on Emerging Technologies and Factory Automation (ETFA)Zaragoza, Spain, pp 1101-1108. https://doi.org/10.1109/ETFA.2019.8869473

Publisher's note Springer Nature remains neutral with regard to jurisdictional claims in published maps and institutional affiliations. 